\title{
Rare Childhood Malignant Neoplasm
}

National Cancer Institute

\section{Source}

National Cancer Institute. Rare Childhood Malignant Neoplasm. NCI Thesaurus. Code C114451.

An infrequent malignant neoplasm that occurs during childhood. 